\title{
A Boilerplate Capsule Test Technique for the Orion Parachute Test Program
}

\author{
James W. Moore* \\ Sierra Nevada Corporation, Louisville, CO, 80027, USA \\ Usbaldo Fraire, Jr. ${ }^{\dagger}$ \\ Jacobs Engineering, Houston, TX, 77058, USA
}

\begin{abstract}
The test program developing parachutes for the Orion/MPCV includes drop tests of a Parachute Test Vehicle designed to emulate the wake of the Orion capsule. Delivery of this test vehicle to the initial velocity, altitude, and orientation required for the test is a difficult problem involving multiple engineering disciplines. The available delivery aircraft options imposed constraints on the test vehicle development and concept of operations. This paper describes the development of this test technique. The engineering challenges include the extraction from an aircraft and separation of two aerodynamically unstable vehicles, one of which must be delivered to a specific orientation with reasonably small rates. The desired attitude is achieved by precisely targeting the separation point using on-board monitoring of the motion. The design of the test vehicle is described. The trajectory simulations and other analyses used to develop this technique and predict the behavior of the test article are reviewed in detail. The application of the technique on several successful drop tests is summarized.
\end{abstract}

\section{Nomenclature}

$\begin{array}{ll}\alpha & \text { Angle-of-attack } \\ C_{D} S & \text { Drag Area } \\ C_{m q} & \text { Pitch damping coefficient }\end{array}$

\section{Introduction}

$\mathrm{T}$ He Capsule Parachute Assembly System (CPAS) project is tasked with developing the parachute system for the Orion/MPCV crew module. CPAS includes a pair of mortar-deployed drogue parachutes intended to decelerate and stabilize the capsule prior to deployment of the main parachute system. The three main parachutes are deployed by a set of three pilot parachutes which themselves are independently mortardeployed. A detailed description of the entire parachute system and concept of operations can be found in Machin, et. al. ${ }^{1}$ The CPAS project has developed a variety of test techniques to evaluate and qualify this system. ${ }^{2}$ The tests described here include the first successful use of a new technique. A critical objective of the test plan is the testing of the deployment of the parachute system from a realistic parachute compartment in a flight-like wake. The test vehicle designed to achieve this goal is a truncated Orion capsule fitted with a hi-fidelity mock-up of the Orion parachute compartment. The options for delivering this type of vehicle to the required altitude are limited. ${ }^{3}$ This paper describes the engineering task of successfully delivering this vehicle to the desired test conditions.

The goals and constraints for the design of the test technique and the resulting Parachute Test Vehicle (PTV) will be described. The PTV is shown in Fig. 1. The vehicle design was subject to significant geometrical constraints that required trade-offs between aerodynamic and structural concerns. The extraction of

*Engineer, Space Systems Group, 1722 Boxelder St., Louisville, CO, AIAA Member.

${ }^{\dagger}$ Analysis Engineer, Aeroscience and Flight Dynamics, 2224 Bay Area Blvd, Houston, TX, AIAA Member. 
the test article from an aircraft required the use of a test support structure called the Cradle and Platform Separation System (CPSS) that acts as an interface between the aircraft and the test vehicle. The test vehicle must be separated from this structure after extraction and before the test point is achieved. The Concept of Operations for this sequence will be described. Design of the trajectory sequence of events and prediction of the test article motion required the development of a new simulation model, enhancement of existing simulations, and new methods for integrating various analysis tools to understand the end-to-end performance of the tests. The toolset used to perform these analyses will be outlined with particular attention to the interface between various tools. The engineers performed a variety of pre-flight analyses to ensure the success of the test. Several of the more important analyses will be outlined. Finally, the deployment of the technique on several tests will be summarized.

\section{History of Test Article Separations on CPAS Tests}

Prior to deploying the PTV test technique, CPAS had developed some experience with separating test vehicles from their carrier platforms. Most of this experience was successful but some key lessons were learned from an unsuccessful test. This section describes the previous experience and how it was applied to lead to the successful PTV tests.

\section{A. Previous Attempt with a Capsule-like Test Vehi- cle}

A boilerplate capsule test had been attempted by CPAS prior to the development of the current PTV. ${ }^{4}$ Lessons learned from the CDT-2 test were critical to the success of the PTV series of tests currently under way. Among other enhancements, the CPAS project developed a Smart Separation system to more precisely control the PTV separation conditions. ${ }^{5}$

\section{B. Hi-fidelity Parachute Compartment Tests}

Prior to the PTV tests, CPAS performed tests with a realistic parachute compartment attached to a dart-shaped vehicle named the Parachute Compartment Drop Test Vehicle (PCDTV). A detailed description of this test technique is provided in Moore. ${ }^{6}$ Prior to the first PTV test, CPAS had successfully deployed the PCDTV on tests CDT-3-1 and CDT3-2. Analysis techniques developed for the PCDTV were also
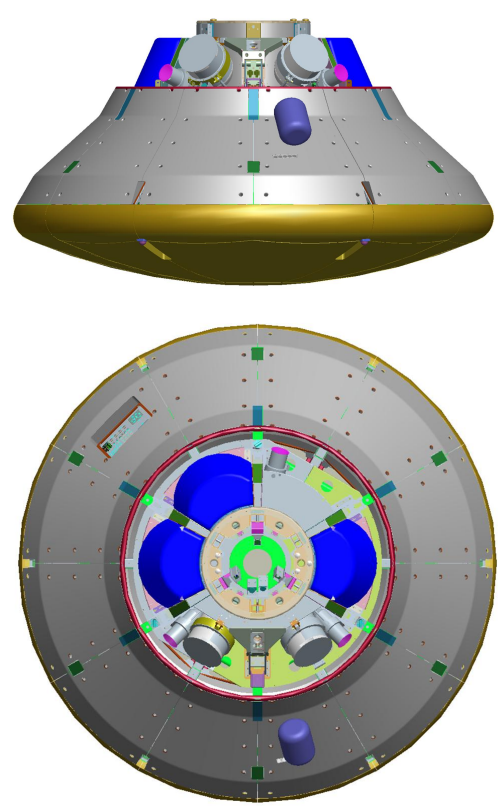

Figure 1. The Parachute Test Vehicle (PTV). adapted to the PTV. For example, the separation of the PCDTV from its extraction carriage was commanded by an avionics package. The release logic employed in the avionics system monitored the PCDTV attitude and rates and commanded release when a desirable attitude is achieved. The definition of the desirable attitude was determined through pre-flight Monte Carlo simulation of various release conditions and assessment of the resulting PCDTV attitude dynamics. This Monte Carlo method was developed for the PCDTV tests which had more predictable dynamics and then adapted to the PTV test series.

\section{Description of the Test Vehicles and Techniques}

The PTV was designed to provide realistic parachute deployments in a flight-like wake. The development of the vehicle and the concept of operations will be described below. Aircraft and structural constraints forced compromises with the aerodynamic goals. The important trade-offs will be highlighted.

\section{A. Concept of Operations}

Figure 2 is a schematic of the PTV test Concept of Operations (ConOps). The mated PTV/CPSS system is extracted from a C-17 using a modified Low Velocity Air Delivery (LVAD) technique with a delayed 
load transfer from the Extraction Force Transfer Coupling (EFTC). The motion of the mated system is monitored by an on-board avionics package. A Smart Separation routine commands the pyrotechnic cut of the lines holding the PTV to the CPSS. CPAS analysts design the separation event to minimize the likelihood of tumbling. The programmer parachute is static-line-deployed from the PTV using a deployment line attached to the CPSS. At a pre-determined time (when predictions show the test point will be achieved) the onboard avionics commands the cut-away of the programmer and the CPAS Drogue parachutes are mortar-fired. After the Drogue parachutes perform the scheduled disreefing, they too are cut and the pilot chutes are mortar-fired. The pilots, in turn, pull out the CPAS Main parachutes. The PTV descends to the ground under the Main parachutes which perform a planned reefing schedule. The parachute cut and mortar-fire events may be based on time or a release algorithm that targets release for the moment of minimum attitude rate. The CPSS descends under the extraction chute system until two CPAS Mains and two stabilizer parachutes are deployed to slow the rate-of-descent to acceptable levels. The stabilizer parachutes are intended to maintain attitude control of the MDS while the Main parachutes inflate. Within this ConOps, a number of test objectives may be achieved. The dynamic pressure and altitude of the Drogue inflation can be modified by selection of the programmer parachute system and programmer cut-away time. The hi-fidelity parachute compartment allows for simulation of the nominal parachute deployment sequence as well as failure scenarios such as a Drogue-out or Main-out condition. The individual parachutes can be rigged to emulate a skipped reefing stage. The PTV will be used to test many of these scenarios leading up to the verification of the system.

\section{B. Test Vehicle Design}

CPAS engineers made several trade-offs in designing the test vehicle. Some of the key constraints are outlined below and the resulting design compromises are described.

\section{Limited aircraft}

Ray $^{3}$ provides a detailed discussion of the potential aircraft capable of delivering an Orion boilerplate capsule to the appropriate test altitude. After consideration of several options, a $\mathrm{C}-17$ was selected as the carrier aircraft and a modified LVAD procedure was chosen to separate the test article from the aircraft. All of the potential test architectures involved some degree of compromise. In the case of the C17/LVAD architecture, the aircraft geometry prohibited the use of a full-scale Orion boilerplate. Rather than uniformally scaling down the boilerplate, the PTV was designed with

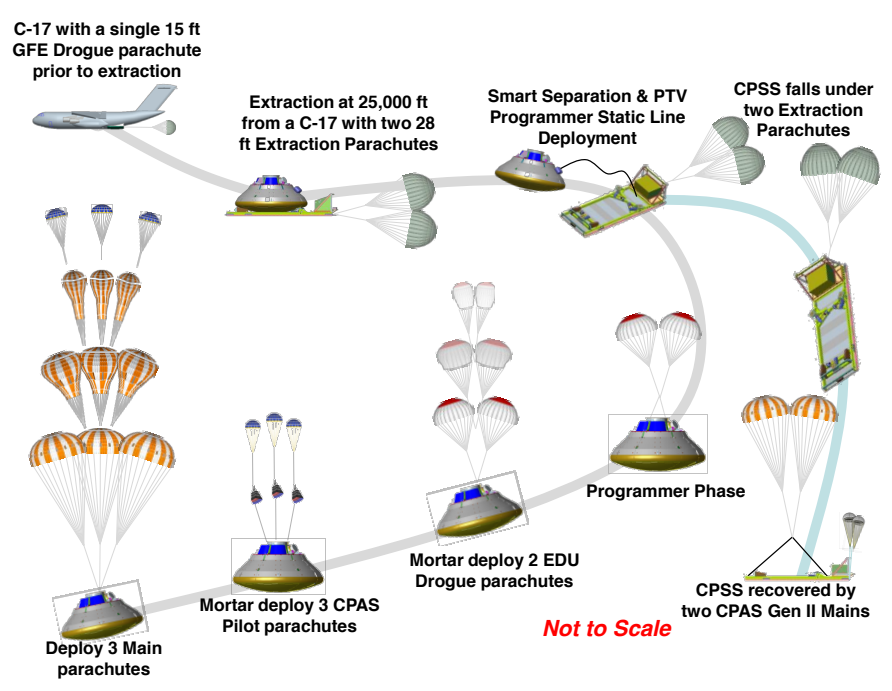

Figure 2. Typical PTV Test Concept of Operations. key components at full-scale but the outer mold line is different than Orion. Figure 3 shows a comparison of the Orion vehicle and the PTV (shown mated to the CPSS).

\section{Differences between the PTV and Orion}

The PTV was designed and built by NASA at the Johnson Space Center. The intent of the vehicle is to provide a parachute test bed with a realistic parachute compartment and a flight-like wake. The PTV diameter at the heatshield is $16.5 \mathrm{ft}$. The mass of the PTV is consistent with current Orion mission configurations. The test program preceded the final design of the Orion parachute compartment but a hi-fidelity mock-up of the parachute compartment makes up top third of the test vehicle. The PTV was designed so that the parachute compartment could be removed and rotated to meet test objectives and will eventually be replaced by a parachute compartment manufactured by the Orion prime contractor. The PTV was significantly shortened relative to the Orion vehicle in order to meet the height constraints of the C-17. To accomplish this, the mid-section of the Orion vehicle is removed from the PTV. This maintains a full-scale 
parachute compartment and heatshield diameter. While the primary objective of the test program is to assess parachute performance, the PTV tests also provide an opportunity to gain insight on the dynamic behavior of the Orion vehicle under various parachute configurations. The need to truncate the PTV alters the inertial characteristics and aerodynamic properties and complicates direct comparisons of the vehicle dynamics.

Another notable difference between the PTV and the Orion is the lack of a Reaction Control System in the PTV. Therefore, the ability to maintain a stable attitude during the test is highly sensitive to the separation conditions and and the effectiveness of the programmer system at damping out inertial and aerodynamic forces. The difference between the aerodynamic properties of the PTV and the Orion vehicle was highlighted in the early tests using this ConOps. This relationship will be described in detail below.

\section{Attach point configurations}

The PTV has 18 attach locations evenly spaced at three levels (6 per level). The attach points are labeled in Fig. 4. These attach points are intended to be used in the programmer phase of the test to allow multiple hang-angles at the test point. The main and drogue parachutes are attached through the parachute compartment as they will be in the operational vehicle. Test engineers are free to select the programmer attach points as needed to achieve test objectives. Multiple attach points are typically used to attach the programmer via a four point harness. Design compromises are required to find an attach point and harness configuration that achieves the desired angle-of-attack at the start of the drogue phase while effectively controlling the dynamics resulting from the PTV/CPSS separation at the start of the programmer phase. The first several tests have settled on a four point harness attached at the

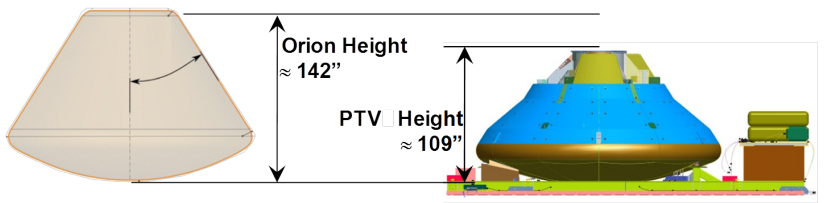

Figure 3. The PTV has the same diameter as Orion but is truncated to allow extraction from a C-17. top of the parachute compartment tunnel with two different lengths of harness legs (two legs at each length) to provide a modest hang-angle. Future tests will employ alternate configurations to achieve a more aggressive angle-of-attack. The tunnel attach points are spaced relatively close together. When coupled with tight constraints on the ballast location levied by stability concerns, the tunnel-attached harness allows a small degree of variation in the PTV hang-angle (and the resulting angle-of-attack). The current mass properties limit the hang-angle to about 20 deg from the vertical. A much broader range of hang-angles can be achieved when the lower attach points are employed. However, simulations have shown such harness configurations to be less effective at arresting the dynamics of the separation event. This continues to be an area of investigation since more aggressive angles are desired for future tests.

\section{Ballast options}

The PTV was designed with space to fix ballast plates in the forward and aft sections near the shoulder of the heat shield (see Fig. 4). In the current configuration, the forward ballast location is fully loaded and the aft location is empty. This moves the center of mass of the PTV as far forward as possible during the extraction and separation phase of the test. Analysis showed that this was the most stable configuration during the separation event. 


\section{Constraint/Release mechanism}

The PTV rests on the CPSS at three locations. The PTV heatshield includes three ball interfaces that rest in respective cups on the CPSS. Prior to separation, the two bodies are secured together with multiple loops of nylon cord that pass through an pyrotechnic cutter. At the desired release point, each of the three cords are cut and allowed to unthread as the PTV separates from the CPSS. Figure 5 shows one of the retention loops and a ball and cup interface.

\section{Test Technique}

The PTV Concept of Operations included two events that had not been successfully performed in previous CPAS tests. Most critically, the successful separation of a boilerplate capsule had not been achieved in the previous attempt. The CPAS project developed a Smart Separation system to target the optimum moment of separation. In addition, the first of the new series of PTV tests showed that the attitude rates at Drogue cutaway could become a critical issue in the more agressive tests planned for the future. A Smart Drogue Release algorithm was

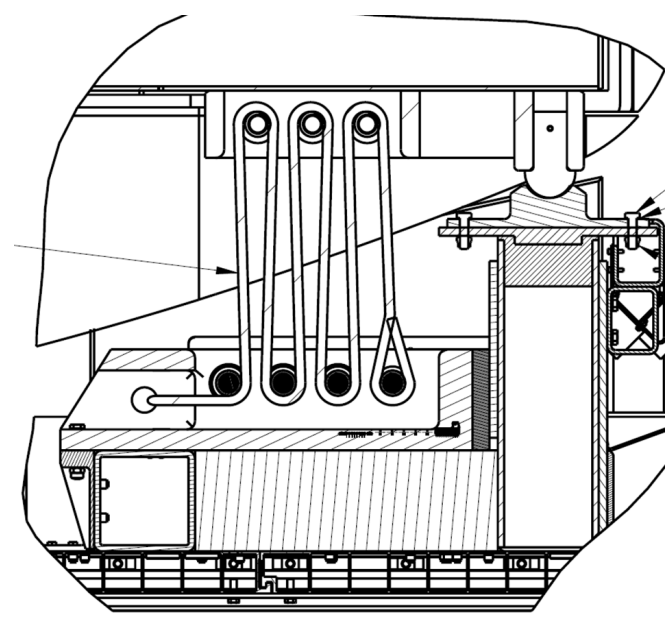

Figure 5. The PTV retention system. The nylon cord (shown by the arrow) is cut to allow the PTV and CPSS to separate. implemented for the second and subsequent PTV tests. The following section provides more detail on the new techniques employed in the the PTV series of tests.

\section{Smart Separation}

The failed PTV test in 2008 used a timed release with the time selected from pre-flight simulation analysis. The uncertainty in the PTV and CPSS aerodynamics and the unpredictable nature of the separation event suggested that a different approach be taken in future tests. Instead of releasing at a predetermined time, the CPAS project developed a system to release within an acceptable range of attitude and rates. The PTV attitude dynamics are highly sensitive to the initial conditions. The aerodynamics of the PTV are such that a significant pitch rate or high angle-of-attack at release can cause the PTV to do a back-flip as the programmer parachute is deploying. Conversely, an insufficient pitch rate or a low angle-of-attack can result in a front-flip. At the time of release, the PTV is in an attitude that would tend to tumble forward. However, it has sufficient pitch rate that it continues to rotate backward toward a heatshield forward orientation. The Smart Separation system was developed to release the PTV at an orientation and rate that will achieve this heatshield forward attitude when the programmer parachute is inflated. The window of acceptable release conditions is only a few tenths of a second long. However, it is difficult to predict when the window will open relative to any other event (for example, clearing the ramp). With Smart Separation, pre-flight simulations can be used to focus on determining the release conditions that tend to achieve the desired attitude rather than predicting the time that this attitude might occur.

The Smart Separation system monitors the pitch and pitch rate of the mated PTV/CPSS and commands separation when both of these parameters have met the predetermined conditions. It also monitors the roll angle of the mated vehicle and can command a release if the roll exceeds a certain deadband. Finally, the Smart Separation logic also has a minimum time before which it will not command separation and a back-up time at which it will command separation regardless of the orientation. The CPAS project developed and refined this technique over several drop tests ${ }^{5}$ including the PCDTV tests that preceded the PTV series of tests. ${ }^{6}$

\section{Smart Drogue Release}

Early tests in the new PTV test series exhibited more oscillation under the Drogues than expected. This led to a concern that future tests meant to model drogue failures would see even more dynamics and possibly result in an undesirable attitude at drogue cut-away and pilot mortar-fire. The Orion Smart Drogue Release logic was adapted to the PTV avionics system and employed on subsequent tests. This system monitors 
the root-sum-square of the pitch and yaw rates and commands the drogue-cut and pilot mortar fire when the rates are at a minimum. Several tunable parameters are included to assist the algorithm in finding an acceptably low orientation rate. A detailed description of the development of this system is provided by Bledsoe. $^{7}$

\section{Modifications Between Tests}

As noted above, engineers observed some unexpected behavior in the first test of the new PTV/CPSS system. The post-flight analysis resulted in changes to the aerodynamic model and hardware changes to improve stability. The postflight analysis and resulting modifications to the test vehicle will be described below. The CDT-3-3 test was the first test of the new PTV/CPSS technique. The separation event occurred as planned and the PTV oscillations damped significantly under the programmer parachutes which are attached by a four point harness to the PTV. The PTV oscillation was excited by the transition from the programmer to the Drogue system which is attached through a single fairlead at a lower point on the PTV. The oscillations were expected to grow under the reefed drogues because of the smaller drag force and the single-point attach. However, the

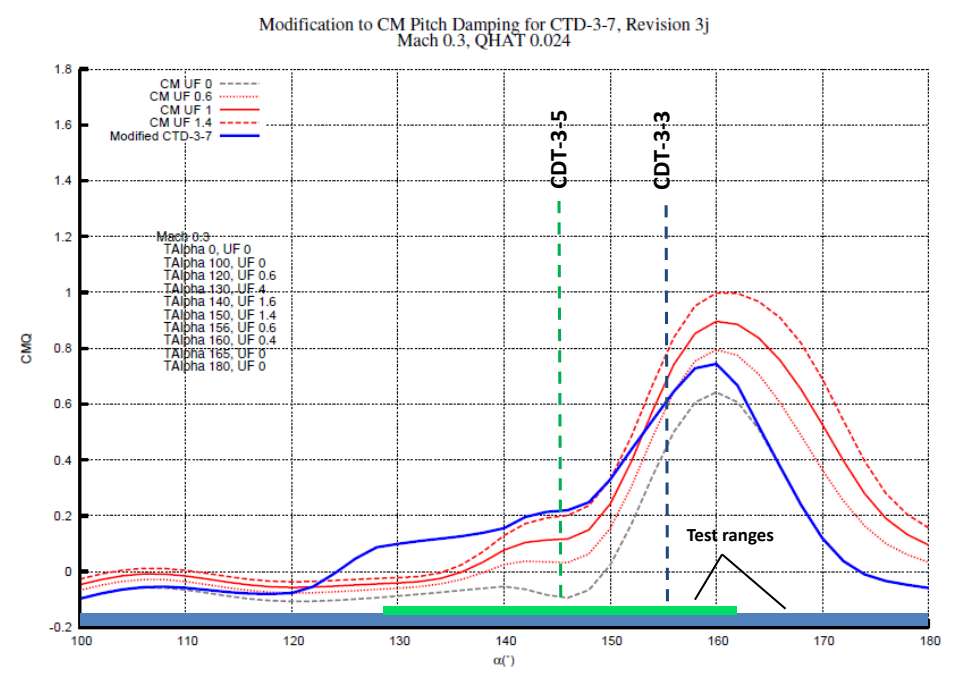
attitude dynamics grew to be larger than anticipated and matched the extreme levels observed in the pre-flight Monte Carlo simulations. The Drogue cut-away occurred at an acceptable attitude and the Main phase was able to stabilize the PTV. The unexpected oscillation was sufficient to cause some concern for future tests and the project made some changes to address the issue.

Three avenues were explored to understand the dynamics and improve performance on the subsequent tests. The first was the addition of the Smart Drogue Release logic described above. The second action was aimed at improving the stability of the PTV for subsequent tests. The PTV ballast is placed in a forward location to improve the stability during the separation event. For CDT-3-3 the parachute compartment was oriented so that the drogue mortars and attach fairlead were also in a forward position. This had the effect of placing the center-of-mass on the same side of the vehicle as the drogue attach point. This configuration was fine for the separation event and programmer phase but was less than optimal for the Drogue phase. The ballast could have been moved to the aft location for future tests but this would decrease stability during the separation event. The PTV cannot be rotated relative to the CPSS to put an aft PTV center-of-mass in a forward mated position. However, the parachute compartment may be rotated relative to the body of the PTV. The CPAS test team elected to leave the ballast where it was and rotate the parachute compartment so that the Drogue attach point and center-of-mass were on opposite sides of the axis of symmetry. This complicated the analysis because the PTV coordinate frame also needed to rotate to use the existing simulations and aero database. This made the relation between the CPSS and PTV coordinate frames as well as the Euler and aerodynamic angles slightly less intuitive. However, the change notably improved the stability under the Drogues for subsequent tests.

The third avenue explored was to better understand and predict the stability of the PTV. The aerodynamic model for the PTV was modified from the full Orion aerodynamic database. Aerodynamically, the PTV looks very similar to Orion for certain angles-of-attack. However, there is a significant difference in the back shell of the two vehicles and at extreme angles of attack (far away from heatshield forward) the vehicles are quite different. Also, the PTV necessarily flies at lower Mach numbers than the actual spacecraft.

Early reconstructions of the CDT-3-3 test indicated that the simulation results would better match the 
test results with a large shift in the pitch damping derivative $\left(C_{m q}\right)$ vs. angle-of-attack $(\alpha)$ curve. Vertical spin tunnel tests of the PTV shape were conducted prior to the PTV drop tests but the results did not warrant a significant change pitch damping derivative as a function of angle-of-attack. Instead, the uncertainty in this factor was increased to encompass the PTV spin tunnel data. This uncertainty level had to be greatly increased to match the actual CDT-3-3 results. Increasing the uncertainty level has the effect of shifting the entire $C_{m q}$ vs. $\alpha$ curve up. Figure 6 shows candidate $C_{m q}$ vs. $\alpha$ profiles and approximate trim angles-ofattack for tests CDT-3-3 and CDT-3-5. To make Monte Carlo predictions for the next test (CDT-3-5), the range of uncertainty in $C_{m q}$ was increased to encompass the instability observed during the drogue phase of CDT-3-3. While a upward shift of the curve provided a rough match of the CDT-3-3 data, the Monte Carlo results for CDT-3-5 included too much uncertainty to provide meaningful predictions. The PTV hang angle under the drogues is such that the angle-of-attack was near the least stable peaks of the $C_{m q}$ vs. $\alpha$ curve. This exaggerated the instability of the PTV for many of the Monte Carlo cycles. To resolve this, a PTV-specific $C_{m q}$ vs. $\alpha$ curve was developed based on motion-matching of the test data. The development of that database is described in Cassidy. ${ }^{8}$ The rotation of the parachute compartment had the effect of moving the trim angle-of-attack away from the most unstable region of the $C_{m q}$ vs. $\alpha$ curve.

In addition to the changes above, improvements were made to the modeling of parachute damping in the simulations. The combined effect of the refinements is a better understanding of the PTV aerodynamics, improved predictive capability, and better stability under the Drogue parachutes. This gave the test team confidence to attempt more aggressive tests such as a single-Drogue test (CDT-3-7).

\section{Toolset Capabilities and Limitations}

CPAS analysts used three simulation to design test trajectories and compute loads during the development of the PTV. The use of multiple trajectory simulations was required because none of the available simulations included all the necessary capabilities. The interface between the various simulations is performed with a suite of MATLAB and Python scripts. Each tool is described here and the strengths and limitations are noted.

\section{A. Decelerator System Simulation (DSS)}

The Decelerator System Simulation ${ }^{9}$ (DSS) is a legacy six degree-of-freedom (6-DOF) parachute trajectory simulation based on the UD233A ${ }^{10}$ simulation used by the Space Shuttle Solid Rocket Booster parachute project. DSS is written in the Fortran programming language and user input is provided via text files. DSS is the highest-fidelity, NASA-maintained simulation used by the CPAS project. However, DSS does not model contact forces between multiple bodies and is, therefore, not suitable for analysis of the extraction phase or the PTV/CPSS separation.

\section{B. Decelerator System Simulation Application (DSSA)}

The Decelerator System Simulation Application ${ }^{11}$ (DSSA) is a 6-DOF parachute trajectory simulation based on DSS that includes the capability to model the extraction of a test platform from an aircraft. Like DSS, this simulation is written in Fortran. All previous CPAS aircraft extractions have been analyzed with DSSA. DSSA does not model the interaction of the vehicles during the separation nor is it well-suited to model the changes in mass properties and aerodynamics that occur when the test article is separated from the CPSS. In addition, DSSA does not necessarily include the all enhancements that have been added to DSS since DSSA became a branch of the main simulation.

\section{Adams}

MSC/Adams is a commercial multi-body 6-DOF simulation. None of the CPAS simulations mentioned above include hi-fidelity multi-body capability suitable for analyzing the separation of the PTV from the CPSS. Adams was applied to address this deficiency. The Adams model developed for these studies included contact forces with the aircraft floor and a simplified extraction parachute drag area, $C_{D} S$, growth curve. This provided significant overlap with DSSA and several sim compare studies were performed to verify the Adams model. A reconstruction of the CDT-2 test was also performed in Adams to provide an additional 
validation step. A hi-fidelity parachute inflation model was not added to Adams so the portion of the test from separation to ground was simulated in DSS.

\section{Load Train Analysis}

Analysts calculated the loads developed during the static-line deployment of the programmer using a set of spreadsheet and MATLAB tools. The spreadsheet methods compute the instantaneous change in velocity at the snatch events and convert this into the energy to be absorbed by the soft goods. The forces are then computed from this absorbed energy result by treating the soft goods as linear springs. Engineers also developed a MATLAB 2DOF deployment simulation that directly integrates the equations of motion for the point masses between the soft goods. This tool was used to crosscheck the spreadsheet results.

\section{Analysis Techniques}

CPAS engineers encountered new problems when developing predictions for the PTV series of tests. In particular, the lack of a hi-fidelity simulation that could model the aircraft extraction, multi-body separation, deployment line loads, parachute inflation, and descent required a patchwork approach to modeling. Several of the new analysis techniques will be described in this section.

\section{A. Coordinate Systems}

A description of the coordinate frames used in the analyses is required in order to understand the upcoming trajectory plots. There are three coordinate

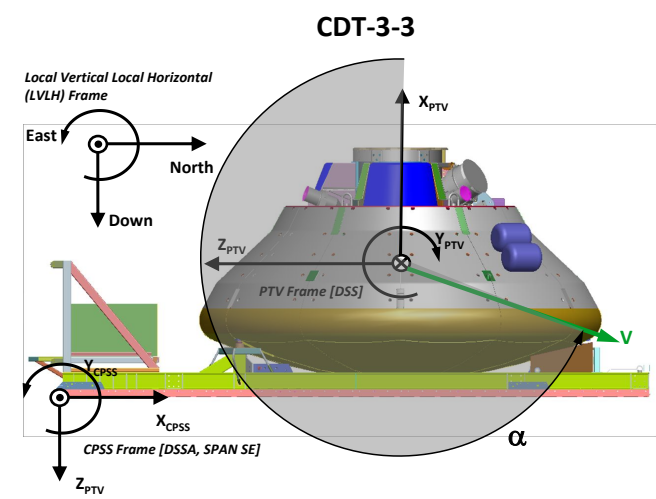

CDT-3-5

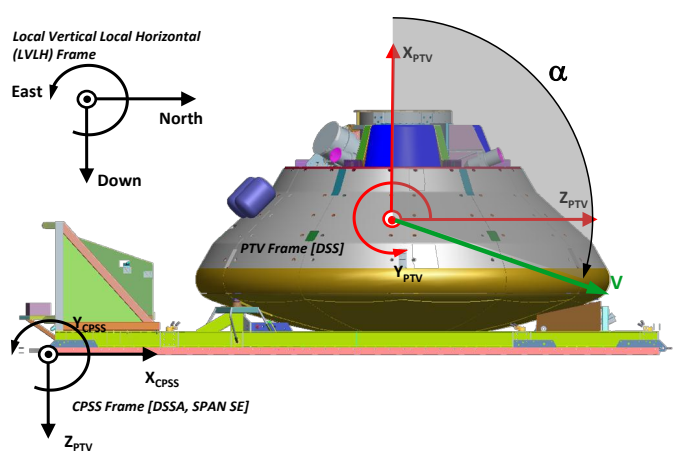

Figure 7. Coordinate frames used in PTV analyses. systems used in the analysis of the extraction and separation of the PTV/CPSS. These are shown in Fig. 7. The multiple systems arise from the use of different 6-DOF simulations for the mated (Adams/DSSA) and un-mated (DSS) portion of the test. The three coordinate systems are a space-fixed Local Vertical Local Horizontal (LVLH) system, a CPSS-fixed body coordinate system and a PTV-fixed body coordinate system. The LVLH frame is common to all simulations and treated as an inertial frame. The coordinate axes are North-East-Down. Yaw-Pitch-Roll Euler angles define the orientation of the CPSS and PTV frames relative to this LVLH frame.

The CPSS frame is fixed to the bottom of the CPSS, centered at the EFTC coupling with z initially pointing down and $\mathrm{x}$ along the platform centerline. The CPSS $\mathrm{y}$-axis points toward the starboard side of the aircraft while the mated CPSS/PTV is onboard. The Smart Separation system was developed and tested on vehicles similar to the CPSS. So the CPSS coordinate frame is used to measure the angles and rates that determine the Smart Separation event. The PTV frame is used for simulations of the PTV after separation so the release conditions need to be transformed from the CPSS to the PTV frame in order to initialize the PTV trajectory analysis.

The PTV frame is centered at the center-of-mass of the PTV with x pointing out through the parachute compartment tunnel. There have been two configurations of the PTV coordinate frame. In order to properly interpret simulation results and compare test data to Orion/MPCV simulations, the PTV frame is necessarily fixed to the parachute compartment (not the PTV forebody). When the parachute compartment was rotated relative to the PTV and CPSS for the CDT-3-5 test, the PTV frame was also rotated 180 deg about the PTV-x axis. Thus, for CDT-3-3 the PTV z-axis pointed parallel but in the opposite direction of the CPSS x-axis. But for CDT-3-5 the PTV z-axis pointed parallel to and in the same direction as the CPSS x-axis, 
both of which pointed toward the direction of flight. The importance of this change in orientation is that the values of the Euler angles and the sense of the angular velocities is different. It was critical to account for this difference when initializing DSS simulations for the second and subsequent tests. Another important distinction is that the angle-of-attack is significantly different as seen in Fig. 7. The DSS simulation defines angle-of-attack as the angle between the body $\mathrm{x}$-axis and the velocity vector measured toward the z-axis. A heatshield forward orientation is therefore a $180 \mathrm{deg}$ angle-of-attack. For simplicity, the plots shown in this paper only use PTV frame orientation associated with CDT-3-3.

\section{B. Identifying Potential Handoff Conditions}

The capabilities of the simulations described above necessitated a hand-off from one simulation to the other at the point of PTV/CPSS separation. The post-separation portion of the test was simulated in DSS. Since there was significant experience with DSS on CPAS, the development of the input deck was fairly straightforward. However, determining the appropriate initial conditions for DSS (i.e. the separation conditions) was complicated because the extraction and separation simulation was not yet developed.

\section{Development of the Phase Plots}

Figures 8 and 9 are examples of key plots that were developed to assess PTV stability. The plot shows the PTV pitch rate vs. angle-of-attack and is similar to a classical phase portrait since angle-of-

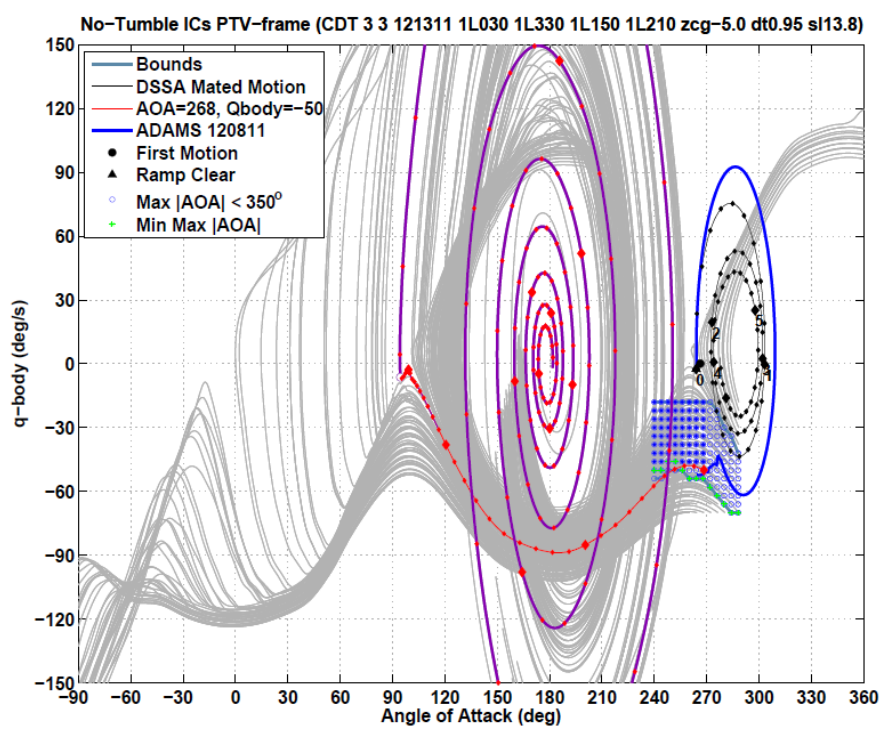

Figure 8. Typical phase portrait for the extraction through programmer phase. attack is related to pitch angle. Angle-ofattack was chosen instead of pitch because it specifies the initial orientation of the vehicle in the DSS sim. In this way the initial conditions that affect the pitch motion are easily plotted on the 'phase portrait'. Several layers of data can be viewed simultaneously to assess the sensitivity of PTV stability to the separation condition. Any $(\mathrm{x}, \mathrm{y})$ point on the plot represents the state of the PTV at an instant of time. As time progresses, the PTV angle-of-attack and pitch rate will follow the line that passes through that point. Lines that spiral towards a single point are cases that damp to a steady state. In this way, these plots can be used to find initial conditions that will damp to a stable condition. Development of an accurate extraction and separation simulation lagged the development of the test phase simulation (programmer through touchdown).

These plots were key to moving forward with the post-separation part of the trajectory planning since they allowed analysts to find candidate separation conditions that the extraction phase must achieve. The separation conditions were varied systematically (without regard to whether the conditions were necessarily achievable) and the resulting trajectories were plotted on the pitch rate, angle-of-attack plane. The postseparation trajectory could then be studied in parallel with analysis of how to achieve the desired separation conditions.

\section{Effects of a Poorly Chosen Release}

Figure 9 shows a close up of the phase portrait near the release condition. The grid between 255 deg and $290 \mathrm{deg}$ angle-of-attack and $-75 \mathrm{deg} / \mathrm{s}$ and $0 \mathrm{deg} / \mathrm{s}$ pitch rate represents sampled initial conditions for the programmer phase of flight. The gray lines emanating from these initial conditions show the trajectory (in phase space) of the PTV as the programmer is deployed. There are two types of trajectories. Some points lead to a increasingly positive pitch rate and an increase in angle-of-attack (up and to the right). Other points lead to an increasingly negative pitch rate and a lower angle-of-attack (down and to the left). In this 
case, the desired outcome is a tight spiral around the point at $180 \mathrm{deg}$ angle-of-attack and $0 \mathrm{deg} / \mathrm{s} \mathrm{pitch}$ rate. A properly chosen release will send the PTV trajectory toward the lower left hand corner of this plot. This will result in a backwards rotation to put the heatshield forward. The upper right portion of the plot represents a forward rotation that puts the apex forward, eventually resulting in a forward flip. A review of Fig. 8 also shows that some release conditions with lower angles-of-attack and larger negative pitch rates do not result in the desired spiral but pass through the desired heat shield forward orientation and complete a back flip.

The dashed line in Fig. 9 represents the motion of the mated vehicle from the time of ramp clear. The achievable release conditions lie on the mated vehicle line. The mated vehicle line is very near the breakpoint in the gray lines that separates the desired outcome from forward tumbles. Significant test configuration changes would be required to control the shape of the dashed line to steer it away from this break point. Instead, analysis focused on finding a range of release conditions that would reliably result in the desired orientation under dispersed conditions. This took the form of repeated Monte Carlo analysis with the Adams and DSS simulations. The Smart Separation system was used to ensure that the release only occurred within this acceptable range.

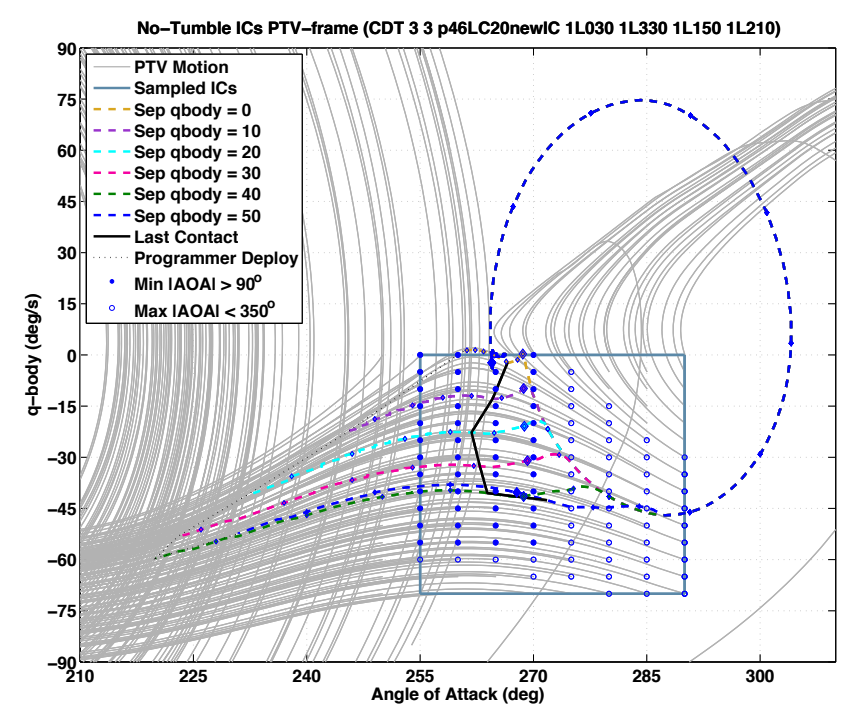

\section{Load Train Analysis}

Figure 9. Zoomed view of a phase portrait to show the separation region.

The loads developed in the deployment train can be significant and must be managed to avoid breaking any of the elements. A failure of the deployment system could result in a delayed or incomplete deployment of the programmer parachutes. Since the programmer parachutes are used to arrest the rotation that follows separation, a failure of the programmer deployment train would likely result in a failed test. Spreadsheet methods were used to compute the changes in velocity of the various mass concentrations within the load train. Energy methods convert these delta-velocities into loads. The load train analysis was also used to estimate the time required to pay out the programmer deployment line. This time was a critical input to the other simulations because it determines the initial conditions for the programmer-to-touchdown phase of the analysis.

\section{Smart Separation Conditions}

As the Adams-based separation simulation began to mature, it became possible to model the extraction and separation of the PTV/CPSS in Adams and hand-off a state vector to DSS to simulate the programmer, Drogue, and Main parachute phases. The phase portrait method described above assisted analysts in understanding the desirable separation conditions and the break points where a suitable solution did not exist. The addition of the Adams model allowed end-to-end simulation of the entire trajectory once release conditions were chosen.

\section{Release Solution}

Figure 10 is an example of how the Adams and DSS simulations were linked together to form an end-to-end simulation and to assess the attitude dynamics resulting from the separation event. The Adams simulation models the extraction and separation portion of the test. This includes the inflation of the extraction parachute, the motion of the mated CPSS/PTV along the aircraft ramp, the pitching motion as the mated vehicle clears the ramp, and the motion of the individual vehicles after the separation command is issued. 


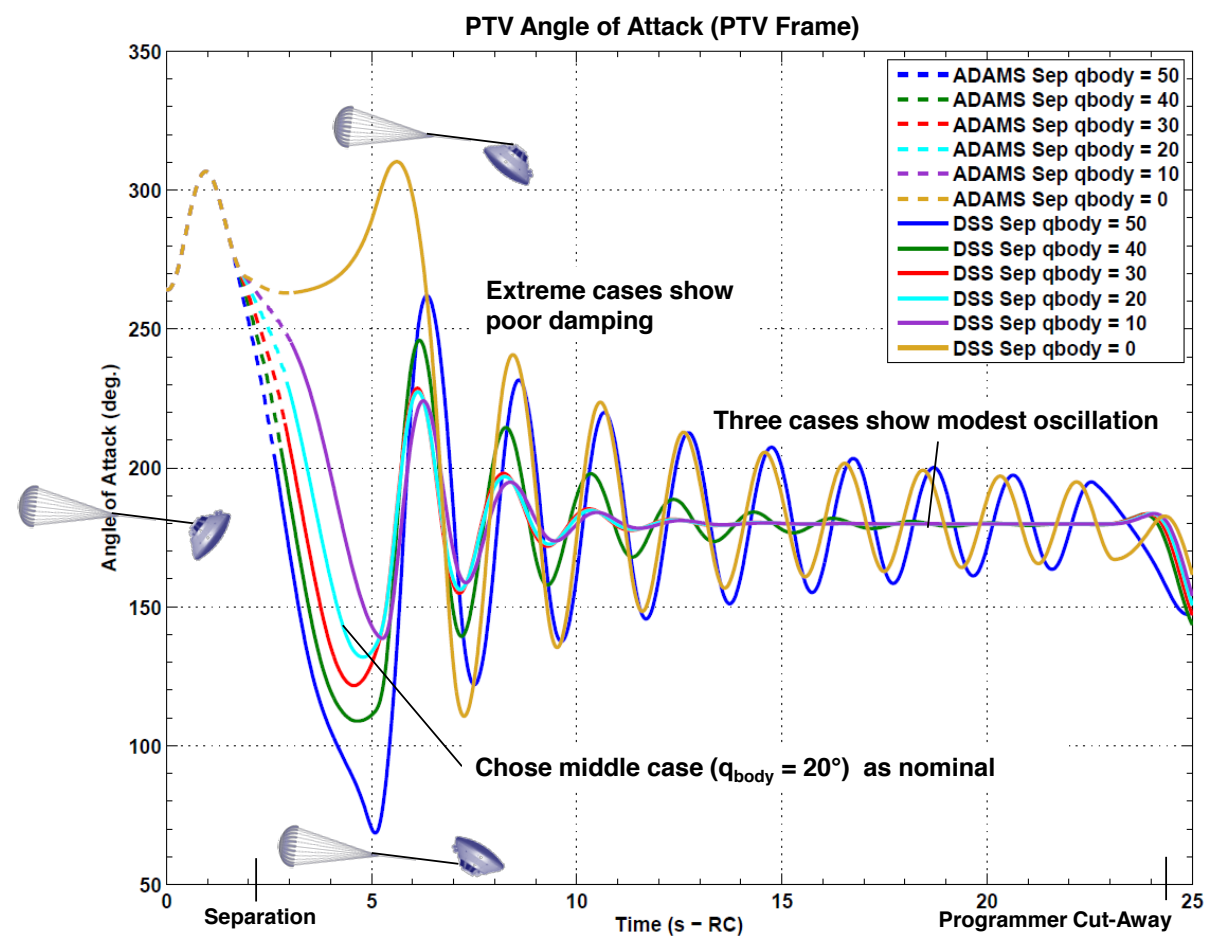

Figure 10. PTV Angle of Attack time history near separation event.

The Angle-of-attack time histories from several Adams simulation runs are plotted as the dotted lines in Fig. 10. The time scale shown is seconds from Ramp Clear ( $-\mathrm{RC}$ ), zero is the instant that the mated vehicle clears the ramp. Each Adams simulation shown in the figure commands release at a different pitch rate. This accounts for the divergence seen at about 3 seconds. The Adams simulation continues until the programmer deployment line has paid out completely. A high fidelity model of the parachute inflation was not developed for the Adams simulation. Therefore, the state of the PTV must be transferred to the DSS simulation at this point. This is repeated for each different release condition.

The various programmer deployment conditions from Adams become initial conditions to DSS and the remainder of the trajectory is computed. The DSS time histories are shown as the solid lines in Fig. 10. Once a target set of conditions was chosen this process was repeated in a Monte Carlo analysis. This required using the output of a set of Adams Monte Carlo simulations to initialize a set of DSS Monte Carlo simulations. The dispersion methods in the two simulations were coordinated. The Smart Separation conditions were refined based on the Monte Carlo results and the process was repeated until the Monte Carlo results were satisfactory.

\section{Time Window Analysis}

In addition to desired conditions on attitude and attitude rate, the Smart Separation system includes a time window. This is a back-up and safety mechanism to prohibit release before a minimum time and to ensure release no later than a maximum time. The analysts considered certain failure modes to assess the appropriate time window. These potential failures included failure to sense a good release condition, erroneously concluding that the release condition was met, and others. The engineers were also aware of the limitations of the modeling and were concerned that the motion may occur faster or slower than the pre-flight simulation indicated. Extensive Monte Carlo analysis was performed on the PCDTV tests to balance the risk of releasing too late (or not at all) with the risk of a sub-optimal release point because the back-up timer was set too early. ${ }^{6}$ A similar analysis was undertaken for the first PTV test but it indicated that the results were highly dependent on the failure mode and that there were few suboptimal (time-based) releases 
that would not result in a tumbling PTV. Back-up release times that would help in one failure mode would make things worse in other failure modes. In the end the project chose a maximum time that was long enough to guarantee a full oscillation of the mated vehicle in order to give the Smart Release system enough time to find the desired release condition assuming no failures. Positive experience on the PCDTV series of tests had built confidence in the performance of the Smart Separation system and the failure modes that the time window was meant to protect against were considered unlikely. On the other hand, the extent of the new modeling added for the PTV series suggested that the pre-flight prediction of the time history of the extraction process was likely to have some modeling error. This risk was considered more likely than an avionics failure so a wide Smart Separation time window was chosen. The minimum time was chosen to ensure safe separation from the aircraft.

\section{Test Results}

The CPAS project has executed three successful drop tests using the PTV. The tests are summarized below.

\section{A. CDT-3-3}

Cluster Development Test 3-3 (CDT-3-3) was the first test of the PTV technique and was designed to test the nominal Drogue, Pilot, and Main sequence at the low end of the dynamic pressure envelope. The separation sequence performed as expected and the PTV was successfully delivered to the test condition. This test marks the first incidence of the Smart Separation system triggering on the optimum pitch and pitch rate release conditions rather than the time-out condition. The aircraft pitch excursion that was experienced on CDT-3-1 and CDT-3-2 during extraction was not present in CDT-3-3 due to the much larger aircraftto-extracted vehicle mass ratio for the C-17/PTV-CPSS combination. The aircraft pitch model had been defined from reconstructed CDT-2 test data. The PTV remained stable following CPSS separation, and as previously discusssed, the Drogue phase exhibited more PTV motion than predicted, but all parachute systems performed nominally. The parachute compartment was rotated for subsequent tests to place the center-of-gravity in a location that would result in more stable Drogue phase dynamics.

\section{B. CDT-3-5}

Cluster Development Test 3-5 (CDT-3-5) was designed to test performance of the system when one of the Main parachutes skips the first reefing stage. The test was performed at the low end of the dynamic pressure envelope with two full-open Drogues as Programmers setting up two Drogues, two Pilots, and three Mains. A reconstruction of extraction was performed by ATK with Adams, resulting in two recommendations. First, the simulation is sensitive to the extraction parachute line of action and should therefore disperse the extraction pitch angle with respect to the horizontal. The pendulum motion of the mated vehicle was matched best when the extraction parachutes were lined up with the aircraft pitch plane, rather than the local velocity vector. This may be due to the aircraft flow field forcing the extraction parachute below the horizontal. The variation in extraction parachute position is shown in Fig. 11

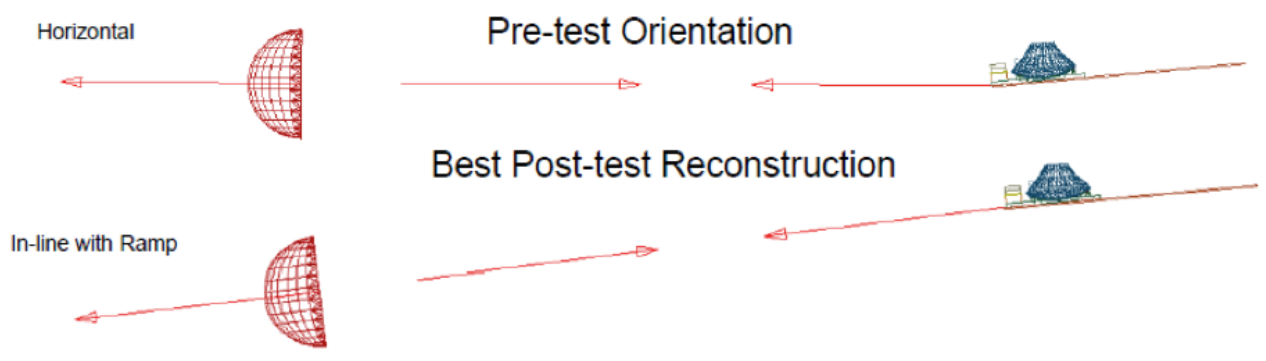

Figure 11. The extraction parachute alignment can affect the pitch motion of the mated vehicle.

The 180-degree rotation of the PTV parachute compartment (and associated hang angle change) resulted in a considerable reduction of PTV dynamics during the drogue phase. Second, it was recommended that 
the short time lag noted between the Smart Separation criteria being met and the determination from video of the retention straps cutting should be introduced into preflight simulations.

\section{CDT-3-7}

Cluster Development Test 3-7 (CDT-3-7) was designed to test performance of the system when a single drogue fails to deploy and when one of the Main parachutes skips the first reefing stage. The test was performed at the low end of the dynamic pressure envelope with one Drogue, three Pilots, and three Mains, set up by two full-open programmers. Final analysis of the data is not complete, but indications are that extraction and separation were nominal and the Drogue phase dynamics were further improved over previous tests with a reduction in aero damping moment uncertainties based on CDT-3-3 and CDT-3-5 test data. The EDU deployment sequence appeared to be nominal.

\section{Conclusion}

The PTV has been successfully employed on three key drop tests. The preliminary design of the separation event required innovation and iteration but the analysis technique has been adaptable to modest changes in the test profile. Design of the extraction and separation sequence can now be performed in a matter of days rather than weeks. Engineers learn more about the dynamic response of the PTV with each drop test and apply lessons learned to subsequent tests.

\section{Acknowledgments}

Keith Anderson of ATK, Pete Cuthbert of NASA, and Lenny Cassidy of Jacobs Engineering performed significant analyses that were instrumental in bringing the PTV technique to test readiness and also made continued improvements to the prediction capability. The authors also wish to thank Ken Nguyen of Jacobs Engineering for supplying many of the diagrams of the PTV used in this paper. There were many other hardware, avionics, and operations engineering tasks that are not discussed in this paper. These challenges were met by the very capable CPAS team who also deserve credit for the success of the tests. Finally, a special thanks goes to Pat Galvin and the CPAS Analysis team for assistance and review in writing this paper.

\section{References}

${ }^{1}$ Machin, R. A., Stewart, C. E., Evans, C. T., and McMichael, J. H., "Human Rating the Orion Parachute System," 21st AIAA Aerodynamics Decelerator Systems Technology Conference, Dublin, Ireland, May 2011, AIAA 2011-2502.

${ }^{2}$ Morris, A. L., Fraire, U., Bledsoe, K. J., Moore, J. W., Olson, L. M., and Ray, E. S., "Simulating New Drop Test Vehicles and Test Techniques for the Orion CEV Parachute Assembly System," 21st AIAA Aerodynamics Decelerator Systems Technology Conference, Dublin, Ireland, May 2011, AIAA 2011-2616.

${ }^{3}$ Ray, E. S. and Morris, A. L., "Challenges of CPAS Flight Testing," 21st AIAA Aerodynamics Decelerator Systems Technology Conference, Dublin, Ireland, May 2011.

${ }^{4}$ Machin, R. A., Stewart, C. E., Evans, C. T., and McMichael, J. H., "Cluster Development Test 2 an Assessment of a Failed Test," 20th AIAA Aerodynamics Decelerator Systems Technology Conference and Seminar, Seattle, Washington, May 2009, AIAA 2009-2902.

${ }^{5}$ Moore, J. W. and Morris, A. L., "Development of a Smart Release Algorithm for Mid-Air Separation of Parachute Test Articles," 21st AIAA Aerodynamics Decelerator Systems Technology Conference, Dublin, Ireland, May 2011, AIAA $2011-2602$.

${ }^{6}$ Moore, J. W., "An Airborne Parachute Compartment Test Bed for the Orion Parachute Test Program," 22nd AIAA Aerodynamics Decelerator Systems Technology Conference, Daytona Beach, Florida, March 2013, Submitted for Publication.

${ }^{7}$ Bledsoe, K. J., "Application of Smart Parachute Release Algorithm to the CPAS Test Architecture," 22nd AIAA Aerodynamics Decelerator Systems Technology Conference, Daytona Beach, Florida, March 2013, Submitted for publication.

${ }^{8}$ Cassidy, L., "Aerodynamic Reconstruction Applied to Parachute Test Vehicle Flight Data Analysis," 22nd AIAA Aerodynamics Decelerator Systems Technology Conference, Daytona Beach, Florida, March 2013, Submitted for publication.

${ }^{9}$ Cuthbert, P. A., "A Software Simulation of Cargo Drop Tests," 17th AIAA Aerodynamic Decelerator Systems Technology Conference, Monterey, California, May 2003, AIAA 2003-2132.

${ }^{10}$ Moog, R. D., Parachute Simulation Userś Guide Computer Program UD233A, Martin Marietta Corp., Denver Aerospace Division, Denver, Colorado, USA, Feb. 1986.

${ }^{11}$ Cuthbert, P. A., "A Desktop Application to Simulate Cargo Drop Tests," 18th AIAA Aerodynamic Decelerator Systems Technology Conference and Seminar, Munich, Germany, May 2005, AIAA 2005-1623. 